\title{
GLOBAL DIMENSION IN NOE'THERIAN RINGS AND RINGS WITH GABRIEL AND KRULL DIMENSION
}

\author{
Juan JaCoBo Simón Pinero
}

\begin{abstract}
In this paper we compute the global dimension of Noetherian rings and rings with Gabriel and Krull dimension by taking a subclass of cyclic madules determined by the Gabriel filtration in the lattice of hereditary torsion theories.
\end{abstract}

\section{Introduction}

In this paper we exhibit a nice subclass of cyclic modules to compute the global dimension of a ring (see [9], [12], [13], [15]) whose origins are in [3] and [11]. In the first part, the left global dimension of a noetherian ring, $R$, is computed in terms of the injective dimerisions of the following subclass of $R$-mod. If $\tau_{-1}<\tau_{0}<\cdots<\tau_{\beta}$ is the Gabriel filtration in the lattice of hereditary torsion theorics of $R$, i.c. $R$-tors [2], then the subclass consist of all the cyclic $\tau_{\mu}$-cocritical left $R$-modules whose injective dimension equals the injective dimension of every one of its submodules, with $\mu$ ranging over all the ordinals less than $\beta$. Also, we obtain some of the classical results for noctherian rings as consequences of our results. In the second part we note that all our results can be dualized.

Throughout this paper, $R$ will denotc an associative ring with 1 and $R$-mod the category of all unitary left $R$-modules. Torsion classes and torsion theories will always be hereditary; all terminology concerning torsion theories is quoted from [2]. Given a nonzero $M \in R$-mod, Id $(M)$ and $\operatorname{Pd}(M)$ denote respectively, the injective and projective dinnensions of $M$, setting $\operatorname{Id}(0)=\operatorname{Pd}(0)=-\infty$. The left global dimersion of $R$ will be denoted by $l g l \operatorname{dim}(R)$, and the Gabriel dimension $G \operatorname{dim}(R)$. For further dctails on cach of these dimensions we refer respectively to [13] and $[2]$. 


\section{Injective dimension}

The Strong Injective Dimension of a left $R$-module, $M$ is defined as $\operatorname{Sid}(M)=\sup \left\{\operatorname{Id}\left(M^{\prime}\right) \mid 0 \rightarrow M^{\prime} \rightarrow M\right.$ is exact $\}$. Following [11], given $n \in \mathbb{N}$, we will denote by $\mathcal{L}_{n}$ the class of left $R$-modules $M$, with $\operatorname{Sid}(M) \leq n$. We define $\operatorname{Sid}(M)=\infty$ when for all $n \in \mathbb{N}$, there exists a submodule $M^{\prime} \subseteq M$ such that $\operatorname{Id}\left(M^{\prime}\right) \geq n$ (with the convention $n<\infty)$. Observe that if there exist $M^{\prime} \subseteq M$ with $\operatorname{Id}\left(M^{\prime}\right)=\infty$, then $\operatorname{Sid}(M)=\infty$. We remark [11] that if $R$ is a left noetherian ring then the classes $\mathcal{L}_{n}(n=0,1, \ldots)$ are torsion classes and [11] if ${ }_{R} R$ is the ring $R$ taken as left $R$-module the $\operatorname{Sid}\left({ }_{R} R\right)=l g l \operatorname{dim}(R)$.

Note that there is a chain of torsion classes $\mathcal{L}_{0} \subseteq \mathcal{L}_{1} \subseteq \cdots \subseteq \mathcal{L}_{n} \subseteq \ldots$ We add $\mathcal{L}_{-\infty}=\{0\}$ and $\mathcal{L}_{\infty}=R$-mod. Let $\sigma_{n}$ be the torsion theory corresponding to $\mathcal{L}_{n}$. Note that $M \in R-\bmod$ is $\sigma_{n}$-torsionfree if and only if for all submodules $0 \neq M^{\prime} \subseteq M$ there exist a submodule $N \subseteq M^{\prime}$ such that $\operatorname{Id}(N)>n$. Within the above chain there exists a strictly increasing subchain; that is, if $n_{0}=-\infty$, then $\mathcal{L}_{n_{0}} \subset \cdots \subset \mathcal{L}_{n_{j}} \subset \ldots$ where the length of the subchain is at most $\omega$.

\subsection{Examples.}

(i) In $\mathbb{Z}$ we have $\mathcal{L}_{-\infty}=\mathcal{L}_{0} \subset \mathcal{L}_{1}=\mathbb{Z}$-mod,

(ii) Let $K$ be a field. For $R=\left(\begin{array}{cc}K & K[X, Y] \\ 0 & K[X, Y]\end{array}\right)$ we have $\mathcal{L}_{-\infty} \subset \mathcal{L}_{0} \subset$ $\mathcal{L}_{1} \subset \mathcal{L}_{2}=R$-mod.

(iii) Let $R$ be a commutative noctherian regular local ring, with $J$ maximal ideal. Suppose that $\operatorname{Id}(R / J)=n$, then we have in this case $\mathcal{L}_{-\infty}=\mathcal{L}_{0}=\cdots=\mathcal{L}_{n-1} \subset \mathcal{L}_{n}=R$-mod.

(iv) Let $R$ be a left artinian left local ring [2]. Then $R$-mod has a chain as in (iii).

(v) In [3], there are examples where all the inclusions in the chain are proper.

(vi) In any left artinian ring which has at least two simple left $R$ modules with different injective dimensions (as $\mathbb{Z}_{12}$ ) the Gabriel filtration has less tcrms than the subchain.

1.2 Lemma. Let $R$ be a left noetherian ring and $\mathcal{L}_{n_{j}}(j=-\infty$, $0,1, \ldots)$ a term in the subchain such that $\mathcal{L}_{n_{j}} \neq R$-mod. Then there exists a $\sigma_{n_{j}}$-cocritical left $R$-module $M$, such that $n_{j}<I d(M)=I d\left(M^{\prime}\right)$ for all submodules $0 \neq M^{\prime} \subseteq M$. 
Proof: Since $R$ is noctherian and $\mathcal{L}_{n_{3}} \neq R$-mod then there exist a $\sigma_{n_{j}}$-cocritical left $R$-module $M$. (a) If $\operatorname{Id}(M)>n_{j}$ (including $\infty$ ) then in each exact sequence $0 \rightarrow M^{\prime} \rightarrow M \rightarrow M^{\prime \prime} \rightarrow 0$ we have $\operatorname{Id}\left(M^{\prime \prime}\right) \leq n_{j}$ and $\operatorname{Id}(M)>n_{j}$, and so $\operatorname{Id}\left(M^{\prime}\right)=\operatorname{Id}(M)$; hence $M$ is the required object. (b) If $\operatorname{Id}(M) \leq n_{j}$ then since $\operatorname{Sid}(M)>n_{j}$ then there exists a submodule $0 \neq M^{\prime} \subset M$ such that $\operatorname{Id}\left(M^{\prime}\right)>n_{j}$ (including $\infty$ ) and since $M^{\prime}$ is also $\sigma_{n_{3}}$-cocritical we are again in case (a), and $M^{\prime}$ is now the required object.

1.3 Proposition. Let $R$ be a left noetherian ring, $\mathcal{L}_{n_{j}}(j=0, \mathrm{I}, \ldots)$ $\mathcal{L}_{n,} \neq R$-mod and $m=\min \left\{I d(C) \mid C\right.$ is cyclic $\sigma_{n,}$, cocritical uith $I d(C)>$ $\left.n_{j}\right\}$. Then $m=n_{j+1}$.

Proof: By hypothesis and Lemma 1.2 it is clear that $\mathcal{L}_{m}$ always exists and that $\mathcal{L}_{n_{j}} \subset \mathcal{L}_{m}$. Suppose that there exists $k \in \mathbb{N}$ such that $\mathcal{L}_{n_{j}} \subset$ $\mathcal{L}_{k} \subseteq \mathcal{L}_{m}$. Since $R$ is noetherian [2] there exists a $\sigma_{n}$-cocritical $\sigma_{k}$ torsion left $R$-module $M$, and hence $n_{j}<\operatorname{Sid}(M) \leq k$. So, by the part (a) in the proof of Lemma 1.2, there exists a cyclic submodule $C \subseteq M$ such that $\operatorname{Id}(C)=\operatorname{Sid}(M)$. Since $C$ is also $a_{n_{j}}$-cocritical and $\operatorname{Id}(C)>n_{j}$. Then, by the definition of $m$ we must have $\operatorname{Id}(C) \geq m$. Hence $k \geq m$ and thus $k=m$.

Note that, in particular, if $m=\min \{\operatorname{Id}(S) \mid S \in R$-mod is simple $\}$ then $m=n_{1}$.

1.4 Observation. Since every subchain has at least two terms, it is natural to analize the step $\sigma_{n_{j}}<\sigma_{n_{j+1}}$. In each of these steps there exists a $\sigma_{n_{j}}$-cocritical cyclic left $R$-module $C$, such that $\operatorname{Id}(C)=\operatorname{Id}\left(C^{\prime}\right)=$ $\operatorname{Sid}(C) \geq n_{j+1}$ for all submodules $0 \neq C^{\prime} \subseteq C$.

From here, Theorem $\mathrm{C}$ of $\mathrm{B}$. Osofsky in [5] follows immediately. In the next theorem, we will sce that we can to extract a nice subclass of the class of cyclic left $R$-modules, to compute the kft global dimension.

1.5 Theorem. Let $R$ be a left noetherian ring, such that $G \operatorname{dim}(R)=$ $\beta$. Then $l g l \operatorname{dim}(R)=\sup \left\{I d(C) \mid C\right.$ is cyclic, $I d(C)=I d\left(C^{\prime}\right)$, for all $0 \neq C^{\prime} \subseteq C$ and $\tau_{\mu-}$-cocritical, with $\left.\mu<\beta\right\}$.

Proof: Let $\tau_{-1}<\tau_{0}<\cdots<\tau_{\beta}$ be the Gabriel filtration in $R$-tors and let $l g l \operatorname{dim}(R)=n_{k}$ (or $\left.\infty\right)$. For any given $j<k$ we have a step $\sigma_{n_{j}}<\sigma_{n_{j+1}}$ and by [2], there exists an ordinal $\alpha \leq \beta$ which is least with the property that $\tau_{\alpha} \& \sigma_{n_{j}}$. Note that $\alpha$ is a successor. Then by Observation 1.4 and the fact that $\tau_{\alpha} \not \sigma_{n_{j}}$ there exists a $\tau_{\alpha-1}$-cocritical $\sigma_{n_{j}}$-cocritical left $R$-moduk $C$, such that $\operatorname{Id}(C)=\operatorname{Id}\left(C^{\prime}\right) \geq n_{j+1}$ for all 
submodules $0 \neq C^{\prime} \subseteq C$. Setting $\mu=\alpha-1$ we have the result in view that the choice of $j$ was arbitrary.

The next corollary is of particular importance inasmuch as there exist an abundancc of examples where the subchain is finite.

1.6 Corollary. Let $R$ be a left noetherian ring such that $G \operatorname{dim}(R)=$ $\beta$. Suppose that we have finitely many terms in the subchain. Then lgl $\operatorname{dim}(R)=\sup \left\{I d(C) \mid C\right.$ is cyclic, $I d(C)=I d\left(C^{\prime}\right\}$ for all $0 \neq C^{\prime} \subseteq C$ and $\tau_{\mu}$-cocritical where $\mu<\beta$ is fixed $\}$.

Proof: Consider the last step in the subchain, $\sigma_{n_{j-1}}<\sigma_{n_{j}}=\chi$. Then there cxists $\alpha \leq \beta$ such that $\tau_{\alpha} \& \sigma_{n_{j-1}}$. Here, $\mu=\alpha-1$.

1.7 Observation. Let $R$ be a commutative noetherian ring. We take in this case the original definition of Krull dimension over the prime ideals of $R$. Suppose that now, for all $S \in R$-rnod simple we have that $S \in \mathcal{L}_{n}$ for some $n \in \mathbb{N}$ (fixed). Let $J$ be any maximal idcal of $R$, then $R / J$ is a simple left $R$-module and $\operatorname{Supp}(R / J)=J$; furthermore, $R / J \in \mathcal{L}_{n_{n}}$. Then, by [1.1] the local ring $R_{J}$ is regular with $K \operatorname{dim}\left(R_{J}\right) \leq n^{2}$. By [13] we have $\operatorname{Sid}_{n_{J}}\left(R_{J}\right)=l g l \operatorname{dim}\left(R_{J}\right) \leq n$, for all $J$. By [13] we have lgl $\operatorname{dirn}(R) \leq n$, hence $R \in \mathcal{L}_{n}$. That is, we have just proved that for any commutative noetherian ring, if $\sigma_{n_{j}} \neq \chi$ then $\tau_{\mathrm{o}} \& \sigma_{n_{j}}$ and by Theorem 1.5 we have the well-known result: $l g l \operatorname{dim}(R)=\sup \{\operatorname{Id}(S) \mid S$ is simple $R$-modulc $\}$.

\section{Projective dimension}

When we compute the left global dimension as the supremum of the projective dimensions of cocritical and critical left $R$-modules we have analogous results to the above; furthermore, we can relax the noetherian condition on the ring $R$. The Strong Projective Dimension [11] is defined as $\operatorname{Spd}(M)=\sup \{\operatorname{Pd}(N) \mid M \rightarrow N \rightarrow 0$ is exact $\}$. We have for all $n \in \mathbb{N}$, the classes $\mathcal{U}_{n}=\{M \in R$ - $\bmod \mid \operatorname{Spd}(M) \leq n\}$. In this case [11] $\mathcal{U}_{n}$ are torsion classes for an arbitrary $\operatorname{ring} R$, and $\lg l \operatorname{dim}(R)=\operatorname{Spd}\left({ }_{R} R\right)$. We denote by $\rho n$ the torsion theory corresponding to $\mathcal{U}_{n}$. Again, we have a chain $\mathcal{U}_{0} \subseteq \mathcal{U}_{1} \subseteq \cdots \subseteq \mathcal{U}_{n} \subseteq \ldots$ and adding $\mathcal{U}_{-\infty}$ and $\mathcal{U}_{\infty}$ we can take a strictly ascending subchain $\mathcal{U}_{n_{0}} \subset \cdots \subset \mathcal{U}_{n_{j}} \subset \ldots$ with $n_{0}=-\infty$. From here, we can do the dualization in a similar way to the first part, and we can remove the noetherian condition. So, we will write only the principal result. For $\rho_{n}$-cocritical left, $R$-modules $C$, such that $\operatorname{Pd}(C) \geq n_{j+1}$, the consequence $\operatorname{Pd}(C)=\operatorname{Pd}\left(C^{\prime}\right)$ for all submodules $0 \neq C^{\prime} \subseteq C$ will be removed in view that all $\rho_{n_{j}}$-cocritical satisfy it. 
2.1 Theorem. Let $R$ be a ring with Gabriel dimension, suppose that $G \operatorname{dim}(R)=\beta$. Then

$$
\text { lgl } \operatorname{dim}(R)=\sup \left\{P d(C) \mid C \text { is cyclic and } \tau_{\mu} \text {-cocritical, with } \mu<\beta\right\} .
$$

2.2 Examples. (i) In a non-noetherian ring $R$, with Gabriel dimension, the classes $\mathcal{L}_{n}$ are not in general torsion classes, but they are Scrre subcategories (see [5]). Even if subchains can be found, the results that we have seen do not hold. For example, let $S$ be $\left(\mathbb{Z}_{2}\right)^{\mathbb{N}}$ and $R \subset S$ the subring generated by $\left(\mathbb{Z}_{2}\right)^{(\mathbb{N})}$ together with $1 \in S$. Then $R$ is a commutative bookan scmiartinian hereditary $V$-ring, having as chain $\mathcal{L}_{-\infty} \subset \mathcal{L}_{0} \subset \mathcal{L}_{1}=R$-mod. Note that the torsion class generated by $\mathcal{L}_{0}$ is the same that $\mathcal{L}_{1}$.

(ii) Let $R$ be a ring with Gabriel dimension and nonsingular as left, $R$-modulc $(Z(n R)=0)$. Then if $R$ is not semi simple we have

$$
\text { lgldim }(R)=\sup \{\operatorname{Pd}(C) \mid C \text { is cyclic singular }\} .
$$

Proof: We shall prove that every $C$ of Theorem 2.1 admits another singular module $D$ such that $\mathrm{Pd}(D) \geq \operatorname{Pd}(C)$. By [4], in every nonsingular ring, cyclic uniform modules are cither singular or nonsingular. Since it is clear when $C$ is singular, we assume that $C$ is nonsingular. So take a left ideal $I$ of $R$ such that $R / I \cong C$. Because $I$ is not large in $R$, there is a left ideal $0 \neq J$ of $R$ with $I \oplus J$ large in $R$. By taking $D=R / I \oplus J$ we have $\operatorname{Pd}(D)=1+\operatorname{Pd}(I \ominus J) \geq 1+\operatorname{Pd}(I) \geq \operatorname{Pd}(C)$ and $R / I$ is singular.

(iv) If $R$ is left semiartinian, $l g l \operatorname{dim}(R)=\sup \{\operatorname{Pd}(S) \mid S$ is simple $\}$ (sce [8]). Semi perfect rings are serniartinian, for instance.

(v) Finally we refer to injective and projective dimension in left Fully Bounded Noetherian (FBN) rings without any other assumption (like the commonly used right coherence of [12], [15]). In this rings, the (two sided) prime ideals often have not hard descriptions and we (ann sce how our classes work.

Let. $R$ be a left. FBN ring and take $\mathcal{L}_{n_{j}} \neq R$-mod $\left(\mathcal{U}_{n_{k}} \neq R\right.$-rnod). By the results above, there exists a cyclic $\sigma_{r_{j}}$-cocritical $\left(\rho_{n_{j}}\right.$-cocritical) left $R$-module $C$. Take [14] the associated prime ideal ass $(C)$ and note that by [14] if $x \in C$ is such that $R \cdot x=C$ then ass $(C) \subseteq l(x)$ (the left amihilator of $x)$ and hence, $R /$ ass $(C) \notin \mathcal{L}_{n_{j}}\left(R / \operatorname{ass}(C) \notin \mathcal{U}_{n_{k}}\right)$ and by [14] we have $R /$ ass $(C)$ is $\sigma_{n_{j}}$-torsionfree $\left(\rho_{n_{k}}\right.$-torsionfrec). Since $R$ is left FBN then, the injective hulls $E(R / a s s(C)) \cong E(C)$ and hence there exists a copy of $C$, say $C$ again, $C \subseteq E(R /$ ass $(C))$. Take $K=$ $C \cap R / \operatorname{ass}(C)$ and note that since $K \subseteq C$ then $K$ has the properties 
of modules in Theorems 1.5 and 2.2. Since $R /$ ass $(C)$ is a left order in a simple ring [7], [14] then for any $x \in K$ we have $(R / \operatorname{ass}(C)) \cdot x \cong$ $R / \operatorname{ass}(C)$ as left $R / \operatorname{ass}(C)$-modules and hence as left $R$-modules. This implies that $\operatorname{Id}(R /$ ass $(C))=\operatorname{Id}(C)(\operatorname{Pd}(R / \operatorname{ass}(C)=\operatorname{Pd}(C))$. So we have that if $R$ is a left FBN ring then

$\lg l \operatorname{dim}(R)=\sup \{\operatorname{Id}(R / I) \mid I \in \operatorname{Spec}(R)\}=\sup \{\operatorname{Pd}(R / I) \mid I \in \operatorname{Spec}(R)\}$.

Note. The author thanks the referee for reporting him about the existence of [6].

\section{References}

1. M.F. ATIYAI AND I.G. MACDONALD, "Introduction to commutative algebra," Addison-Wesley, Reading, Mass., 1969.

2. J.S. GoLAr, "Torsion theories," Longman Scientific \& Technical John Wilcy \& Sons, 1986.

3. J.S. GOLAN AND Z. PAPP, Cocritically nice rings and Boyle's conjecture, Comm. Algebra 8(18) (1980), 1775-1798.

4. K.R. Goodearl, "Ring Theory," Marcel Dekker, New York, 1976.

5. R. GORDON AND J.C. RoBson; Krull dimension, Mem. Amer. Math. Soc. 133 (1973).

6. J.J. KoKER, Homological dimension of rings with Krull and Gabriel dimension, Ph.D. Thesis, University of Wisconsin-Milwaukee, 1990.

7. J.C. MC CONNELL AND J.C. ROBSON, "Noncommutative noetherian rings," John Wilcy \& Sons, Chichester New York Brisbane Toronto Singapore, 1987.

8. C. \ЙS'TĂSESCU, Dimension globale des anneaux semi-artiniens, C.R. Acad. Sci. Paris, Sér. A-B 268 (1969), A685-A688.

9. B.L. Osorsky, Global dimension of valuation rings, Trans. Amer. Math. Soc. 127 (1967), 136-149.

10. I. PALmér AND J.-E. Roos, Formules explicites pour la dimension homologique des anneaux de matrices generalisées, C.R. Acad. Sci. Paris, Sér. A-B 273 (1971), A1026-A1029.

11. Z. PAPP, On the strong injective (projective) dimension of modules, Arch. Math. 25 (1974), 354-360.

12. J. RAINWATER, Global dimension of filly bounded noetherian rings, Comm. Algebra 15(10) (1987), 2146-2156.

13. J. ROTMAN, "An introduction to Homological Algebra." Orlando, Academic Press, 1979. 
14. Bo Stenström, "Rizigs of quotients," Springer-Verlag, Berlin, Heidelberg, New York, 1975.

15. M. TEPLY, Global dimensions of right coherent rings with left Krull dimension, Bull. Austral. Math. Soc. 39, no 2 (1989), 215-223.

Departamento de Matemáticas

Universidad de Murcia

30071 Murcia

SPAIN

Primera versió rebuda el 8 de Juliol de 1991 , darrera versió rebuda el 15 d'Octubte de 1991 